\title{
Systematic prioritization of cancer combination therapies: are we really on target?
}

"...the toxic side effects of targeted agents have proven to be more significant than originally theorized. In the context of the high cost of targeted agent regimens and the unexpectedly high rate of severe side effects, the risk-cost-benefit equation becomes increasingly hard to solve..."

Keywords: combination $\|$ companion diagnostics $\|$ genomics $\approx$ multi-targeted $\approx$ personalized medicine $\approx$ synergy

What happened to the promise of the 'magic bullet' in oncology? In the 1990s there was an explosion of genomic approaches to identify new drug targets in cancer - sequencing, expression profiling and functional cloning, all designed to identify new genes to feed the pharmaceutical industry's target-based drug-discovery engine. A plethora of extremely potent and highly selective targeted agents emerged from these efforts only to see unexpected failures of efficacy when used as monotherapies. The focus then shifted to pharmacogenomics and identification of specific subpopulations whose genomic profile could enrich therapeutic responses to targeted agents. Yet the effective realization of this approach has been plagued with numerous technical and regulatory challenges limiting its application to a handful of examples [1,2]. Thus, two decades later, the pharmaceutical industry has invested billions of dollars into the discovery and development of highly targeted agents and large consortia have embarked on huge efforts to catalog the genomic profile of tumor cell lines and patient samples. Yet the standard of care in most oncology indications is still centered around combinations of cytotoxic agents with limited guidance based on genomic or biomarker diagnostics.

To be sure, there has been progress in identifying selective targeted agents that when deployed in the right patient populations give remarkable results. All cancer researchers know the gold standard examples: Gleevec ${ }^{\circledR}$ in Philadelphia chromosome-positive chronic myelogenous leukemia [3]; Herceptin ${ }^{\circledR}$ in HER2-positive breast cancer [4]; Iressa ${ }^{\mathrm{TM}}$ and Tarceva $^{\mathrm{TM}}$ in EGFR-positive lung cancer $[5,6]$; and Xalkori ${ }^{\circledR}$ in lung cancer harboring ALK fusions [7]. These drugs have served as models to assess the power of a specifically targeted agent in a carefully selected patient population to demonstrate substantial response rates and, in many cases, survival benefit. Rituxan ${ }^{\circledR}$, one of the first monoclonal antibodies approved in oncology, has demonstrated clear benefit in terms of response rates and progression-free survival in several B-cell malignancies and data from long-term follow-up studies suggests that Rituxan-containing regimens also confer survival benefit [8]. Other targeted agents such as Avastin ${ }^{\circledast}$ have seen some controversy in their use, especially in breast cancer where no clear survival benefit has been demonstrated [9]. Refractory disease and emergence of resistance mechanisms continue to be real challenges associated with highly targeted agents. Furthermore, the toxic side effects of targeted agents have proven to be more significant than originally theorized. In the context of the high cost of targeted agent regimens and the unexpectedly high rate of severe side effects, the risk-cost-benefit equation becomes increasingly hard to solve, especially in cases where long-term randomized trials show mere weeks of additional survival benefit. When compared with the level of investment made by our industry, these results are far from the original promise offered by the genomic revolution and the advent of personalized medicine.

What are the challenges that have conspired to limit our success despite the concerted efforts and investments of pharmaceutical, biotechnology and academic consortia? The first and most obvious is the complexity of cancer biology. Efforts to profile and catalog the driver mutations and pathways responsible for cancer proliferation

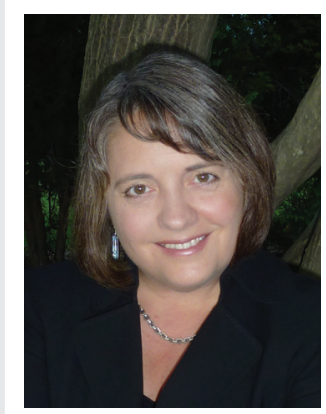

Margaret S Lee

Zalicus Inc., 245 First St, 3rd Floor, Cambridge, MA 02142, USA

E-mail: mlee@combinatorx.com 
and metastasis and integrate information such as mutational analysis, copy number, geneexpression changes, metabolic profiling and proteomics underscore the enormous number of candidate pathways and changes that may be responsible. This, coupled with powerful inherent mechanisms of redundancy, the ability to adapt and compensate, and the emergence of resistance, unite to circumvent the impact of any single antineoplastic insult. In hindsight, it seems obvious that a complex polygenic disease such as cancer, would be refractory to the single magic bullet approach. Yet despite this growing evidence, our industry has been slow to react in shifting away from the comfort of generating ever more potent and selective highly targeted agents based on rational drug design. The focus on 'clean' highly selective drugs over 'dirty' multitargeted agents has led us away from efficacy in monotherapy. To restore the complexity of the multi-targeted therapy, the empirical evaluation of effective combinations of targeted agents in the clinic has become a focus. However, this approach suffers many challenges, not the least of which is the slow and expensive process of testing combinations in multi-arm randomized clinical trials. This, coupled with an increasingly conservative regulatory environment, the uncertain registration path associated with co-development of more than one new chemical entity in combination, the need for effective companion diagnostics and limited enthusiasm of individual companies to pair agents outside of their proprietary pipeline, have presented great hurdles to the successful development of effective multi-targeted combinations [1,2].

"... industry has been slow to react in shifting away from the comfort of generating ever more potent and selective highly targeted agents based on rational drug design."

What is needed is clear: rapid and efficient ways to identify and prioritize multi-targeted therapies that confer selective tumor cell death with limited toxicity in highly defined and easily identified patient populations. There is a general consensus in the industry that this synergy and selectivity can be achieved through combinations. As evidence of this are the many effective combination regimens developed in oncology to date, and even more profound success with combinations of targeted agents is seen in other indications such as infectious diseases. These effective combinations have been developed over decades of study in the clinic, some based on rational hypothesis-driven approaches and others by trial and error. With so many targeted agents and oncology drugs to choose from, how do we rapidly and efficiently identify the best combinations, define the patient populations in which they will be effective and deploy them in the clinic? The answer lies in the systematic and empirical evaluation of all possible combinations of known drugs and drugable targets across many disease-relevant cancer cell line models with defined genomic profiles. This differs from the many small-scale, hypothesis-driven combination experiments reported from both industrial and academic laboratories. To fully exploit complex tumor biology and most effectively deploy the many potent targeted agents available, a truly agnostic screening approach is required. For example, assuming there are approximately 500 relevant cancer targets, the evaluation of all possible unique pair-wise combinations results in nearly 125,000 possible tests. When considering that many new combination therapies will be added on to standard-of-care regimens the number of possibilities quickly moves into the millions. Including assessment of multiple doses and ratios and evaluation across multiple tumor cell line models further enlarges the complexity of combination space to be explored. Thus, the richness of possibility in this space rivals our industry's best efforts to generate complexity through combinatorial chemistry approaches. Furthermore, when driven by the empirical observation of synergistic efficacy, this approach leverages the complexity of tumor biology as an ally in screening. The universal application of this approach across the many hundreds of relevant tumor cell line models available represents the most systematic and empirical application, while focused screening across a subset of these models in a particular indication (e.g., breast cancer) would effectively limit resource utilization while maintaining an agnostic approach. The translational potential of the resulting combinations could be even further enhanced by the addition of primary or secondary screening assays with improved predictive validity (e.g., ex vivo patient samples, complex models of the tumor microenvironment).

The successful clinical deployment of this approach requires the development of companion diagnostics designed to detect the complex genomic environment where a given combination will be most effective. This 
differs from the traditional approach taken by most cancer-focused diagnostic firms where diagnostic portfolios are based on emerging or clinically validated targeted therapies. In contrast, the focus would be on identifying the set of genomic determinants that define sensitivity or resistance to the synergistic effect conferred by a combination of drugs or targets. The end product would be a test for a set of genomic determinants that, when present in the same tumor, would predict greater sensitivity to a particular multi-targeted anti-tumor insult. At face value this seems challenging. Indeed, if we have had limited success as an industry in defining the genomic signature of sensitivity and resistance to single agents how then could this be accomplished effectively with combinations? Here is where the power of combination biology can be used to our advantage. The synergistic effects introduced through multitargeted combinations will be more effective at revealing the innate differences in redundant or interconnected biological networks than any single anti-tumor intervention. By systematically probing these innate differences with combinations of drugs and targets, greater selectivity can be revealed, resulting in large differences in efficacy among tumor models with different underlying pathological networks. By focusing on synergistic anti-tumor combinations that demonstrate substantial differential effects across large panels of tumor cell line models, the differences in genomic signature between sensitive and resistant populations can be more effectively exploited.

Never before has our industry been better positioned to embark on this approach. Recent efforts by academic and industry consortia, such as The Cancer Genome Atlas [101], the Broad-Novartis Cancer Cell Line Encyclopedia [102] and the Wellcome Trust Sanger Institute Cancer Genome Project [103], have made publicly available large databases of detailed genomic information cataloging genetic, epigenetic and proteomic alterations that are rigorously associated with particular human tumor types and cancer cell lines. A mature and efficient high-throughput combination screening-andanalysis platform is currently operating in the author's laboratory that has proven ability to tackle projects of this scale. Diagnostic technologies have evolved to meet the challenge of evaluating patient samples on a time scale useful to clinical decision making. What remains is the willingness of our industry to invest in this approach and the discipline required to define the regulatory path forward for combination therapeutics with companion diagnostics. We are poised to identify highly synergistic and selective combinations, paired with well-defined diagnostics for genomic signatures of response, enabling the rapid clinical evaluation of the most effective combinations. Embracing this approach brings us the possibility of fulfilling the promise to provide truly meaningful clinical benefit to cancer patients.

\section{Financial \& competing interests disclosure}

The author is employed by Zalicus Inc. and owns stock options in Zalicus Inc., which performs combination highthroughput screening. The author has no other relevant affiliations or financial involvement with any organization or entity with a financial interest in or financial conflict with the subject matter or materials discussed in the manuscript apart from those disclosed. No writing assistance was utilized in the production of this manuscript.

\section{References}

1 Gonzalez-Angulo AM, Hennessy BT, Mills GB. Future of personalized medicine in oncology: a systems biology approach. J. Clin. Oncol. 28(16), 2777-2783 (2010).

2 Philip R, Carrington L, Chan M. US FDA perspective on challenges in co-developing in vitro companion diagnostics and targeted cancer therapeutics. Bioanalysis 3(4), 383-389 (2011).

3 Ren R. Mechanisms of BCR-ABL in the pathogenesis of chronic myelogenous leukaemia. Nat. Rev. Cancer 5(3), 172-183 (2005).

4 Brufsky A. Trastuzumab-based therapy for patients with HER2-positive breast cancer: from early scientific development to foundation of care. Am. J. Clin. Oncol. 33(2), 186-195 (2010).

5 Laack E, Sauter G, Bokemeyer C. Lessons learnt from gefitinib and erlotinib: key insights into small-molecule EGFR-targeted kinase inhibitors in non-small cell lung cancer. Lung Cancer 69(3), 259-264 (2010).

6 Siegel-Lakhai WS, Beijnen JH, Schellens JH. Current knowledge and future directions of the selective epidermal growth factor receptor inhibitors erlotinib (Tarceva ${ }^{\mathrm{TM}}$ ) and gefitinib (Iressa $^{\mathrm{TM}}$ ). Oncologist 10(8), 579-589 (2005).

7 Shaw AT, Yasothan U, Kirkpatrick P, Crizotinib. Nat. Rev. Drug Discov. 10(12), 897-898 (2011).

8 Keating GM. Rituximab: a review of its use in chronic lymphocytic leukaemia, low-grade or follicular lymphoma and diffuse large B-cell lymphoma. Drugs 70(11), 1445-1476 (2010).

9 Dienstmann R, Ades F, Saini KS, MetzgerFilho O. Benefit-risk assessment of bevacizumab in the treatment of breast cancer. Drug Saf. 35(1), 15-25 (2012).

\section{- Websites}

101 The Cancer Genome Atlas. http://cancergenome.nih.gov

102 Broad-Novartis Cancer Cell Line Encyclopedia. www.broadinstitute.org/ccle/home

103 Wellcome Trust Sanger Institute Cancer Genome Project. www.sanger.ac.uk/research/projects/ cancergenome 